\title{
Laser Synthesis of Gold Nanostructures at Atmospheric Pressure: Nature and Applications
}

\author{
K. V. Kozadaev \\ A.N. Sevchenko Scientific-Research Institute of Applied Physical Problems of Belarussian State University, \\ Minsk, Belarus \\ e-mail: kozadaeff@mail.ru \\ Received December 4, 2013
}

\begin{abstract}
Consideration has been given to certain aspects of condensation processes occurring in laser-induced erosion plumes of gold and giving rise to fluxes of golden nanoparticle at atmospheric pressure. Samples of colloidal solutions of gold and gold-containing polymer films have been produced as a result of penetration of nanoobjects formed in erosion plume of gold into aqueous and polymer-containing media. On the base of air laser synthesis it can be also possible to obtain the gold surface island-shaped nanostructures in the form of nanocones widely distributed with height. Experimental samples formed have been comprehensively investigated using optical spectroscopy, atomicforce microscopy, transmission and scanning electron microscopy.
\end{abstract}

Keywords: laser synthesis, gold nanoparticles, colloidal solution of gold, gold-containing, polymer film, surface plasmon resonance, nanocones of gold

DOI: $10.3103 / \mathrm{S} 1060992 X 14010019$

\section{INTRODUCTION}

The increasing practical interest currently expressed in methods of formation and investigation of isolated metal nanoparticles is due to the wide range of advantages which are offered by traditional media modified by metallic nanostructures. Ultra-disperse metal-containing media may exhibit specific electrical, magnetic, optical, thermophysical, chemical, biological, and other properties [1-3]. The mentioned above features are determined by the presence of the so-called dimensional effects for isolated metal particles with a diameter less than $100 \mathrm{~nm}$. A large number of technologies of producing metal nanoparticles have been developed; they may be divided into the following groups: elementary wet chemistry with the use of deposition and reduction of metals, synthesis of nanoparticles from the gas and solid phases, chemical homogenization, production of nanoparticles in one-dimensional and two-dimensional reactors, synthesis with the use of such phenomena as self-organization and self-assembly, various kinds of lithography [2]. The enumerated methods demonstrate their own advantages and drawbacks and can be applied to the solution of certain technological problems.

Specific properties of gold as a metal (chemical stability, inertia, relative infusibility, and high specific weight) make it impossible to use in proper way most of the existing methods of nanostructures production. Chemical methods traditionally used for these purposes are rather resource-intensive and expensive [4]. As an alternative approach we can consider the technique of laser synthesis of gold nanostructures [5]; this technique is based on the experimental fact that under laser erosion of gold targets by intensive nanosecond pulses at air conditions the two-phase plasma fluxes containing gold nanostructures can be obtained [6]. The use of modern frequency-modulated lasers for production of gold-containing media makes it possible to improve the competitiveness of the procedure of laser synthesis compared to other techniques of formation of ultra disperse metal-containing media. Additional advantages of the indicated technique are the technological simplicity of the synthesis plant, the possibility of controlling the process of synthesis on line, and the high rates of formation of colloids [7].

\section{THEORY}

Character of Gold Targets Destruction by Nanosecond Laser Pulses

According to classic theory in the process of exposure of massive gold planes to intense nanosecond laser pulses in a thin near-surface region of the target, part of the irradiation is absorbed by free conduction 
(a)

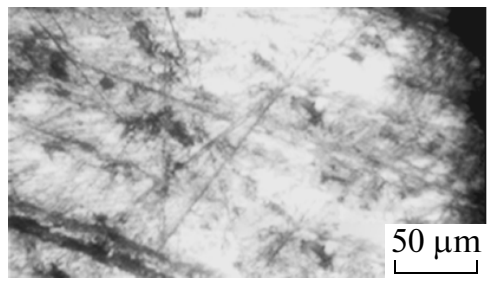

(b)

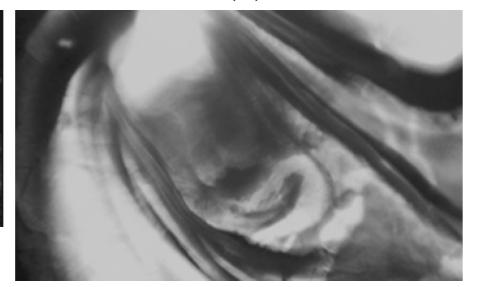

(c)

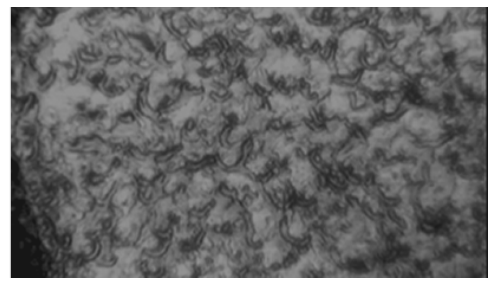

Fig. 1. Relief of the metal target: (a) initial, (b) after acting of powerful sub-millisecond pulse, (c) after acting of powerful nanosecond pulse.

electrons [8] in thin surface layer $(\sim 1 \mu \mathrm{m})$. It should be noted that the initially high coefficient of reflection of optical radiation by gold surface $(R=0.92$ for a light wavelength of $1000 \mathrm{~nm}$ [9]) under intense laser action can sharply decrease, enhancing the integral dose of pulse energy absorbed by the target to $50 \%$ of the total optical energy reaching the target surface [10]. In connection with the fact that in case of nanosecond pulse (even with duration $\sim 10 \mathrm{~ns}$ ) implementation the leading-edge time of laser pulse $\left(10^{-9} \mathrm{~s}\right)$ becomes comparable with the characteristic time of electron-ion thermal relaxation in metals $\left(>10^{-10} \mathrm{~s}\right)$ [8]. So the absorbed optical energy can not penetrate deep into the target material due to only electronic thermal conductivity, therefore the role of irradiative thermal conductivity significantly increases. As a result a macro-layer of the "solid-vapor" phase transition virtually without the formation of a liquid phase is formed under the influence of excess energy in the thin $(\sim 5-10 \mu \mathrm{m})$ surface layer of the target [10]. This macro-layer can be comparable with an explosive layer, which detonation process is followed by two basic physical effects: formation of the metal vapor-plasma plume (rapidly expands to outer space) and generation of the intensive shock wave (propagates deep into the target). This model of laser erosion of metals is called a "hydrodynamic" model [8], since it is precisely the hydrodynamic plasma motion that determines the basic regularities of interaction.

At the duration of the front of rise of the pulse intensity of 5-10 ns, the vapor-plasma plume formation is generated with a delay of 10-20 ns from the beginning of exposure, suggesting that the radiation of the entire, in practice, leading edge of the pulse reaches the target surface without losses [11]. At the initial stage of its formation the vapor-plasma plume (due to the inverse breaking effect) begins to actively absorb the optical energy of the recession edge of the acting laser pulse, increasing its internal energy owing to this and rapidly propagating in the atmosphere (according to the evaluations of [10] the initial velocity of vapor-plasma plume of gold is $4 \mathrm{~km} / \mathrm{s}$ ). After the finishing of the laser pulse, adiabatic expansion of the vapor-plasma plume continues, which leads to its gradual cooling. In the vapor-plasma cloud, there appear local density fluctuations which subsequently turn to drop-liquid particles of the target material due to the condensation processes. The result average sizes of gold particles lie in the nanometric range $(30-50 \mathrm{~nm})$ and vary depending on the exposure conditions. This condensed phase of metal in the form of nanoparticles is present in the surface region of the target over a rather long period: $500-600 \mu \mathrm{s}$ from the beginning of exposure to the laser pulse [6].

The described process of laser erosion is accompanied by the appearance of characteristic changes in the relief in the form of sinuous microchannels (with transverse dimensions of the order of several microns and longitudinal dimensions of the order of tens of microns) on the target surface (Fig. 1c). For a comparison at the Fig. 1 the initial relief of metal target (a) and the result of powerful sub-millisecond pulse action (b) are shown.

The investigations carried out have shown that the initial relief of the target surface significantly influences the efficiency of formation of the condensed phase of gold [6]. Substantial roughness on the target surface (in the form of longitudinal scratches with transverse dimensions of $\sim 50-100 \mu \mathrm{m}$ ) increase the concentration of nanoparticles in the erosion plume by 2 to 3 orders (up to $10^{12} \mathrm{~cm}^{-3}$ ) of magnitude compared to the smooth target surface (with irregularities of $\sim 1-3 \mu \mathrm{m}$ ). This effect is attributable to the increase in the effective interaction area of the rough surfaces and to the presence of numerous gas-formation centers.

In studying the influence of the factor of the target's relief on the efficiency of the process of laser synthesis of nanoparticles, in the case of multiple laser action without changing the localization of the focal spot we have observed the effect of a decrease in the concentration of metallic nanoparticles in the erosion plume for each subsequent pulse. After 4-5 actions of this kind, the efficiency of nanoparticle formation is no different from the case of a smooth target irrespective of the initial level of roughness of the target. 
(a)

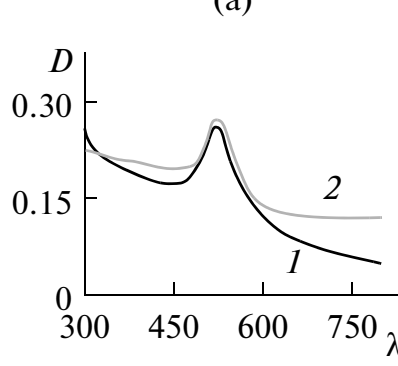

(b)

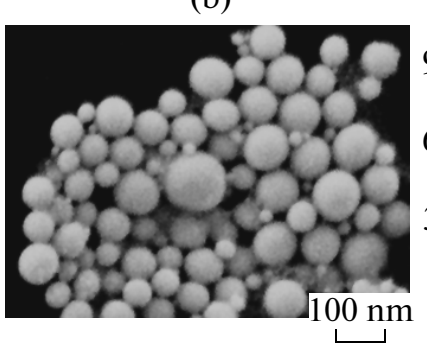

(c)

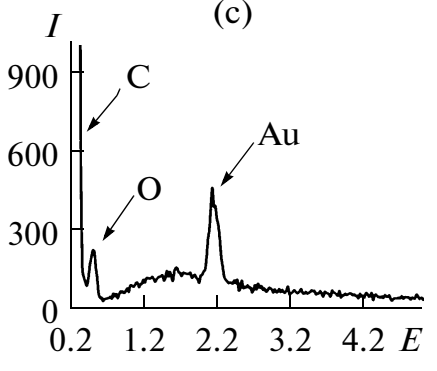

Fig. 2. The results of golden nanoparticles investigation: (a) SPR band spectra of gold colloid (1-real, 2-model), (b) SEM image of golden particles deposited on a $\mathrm{C}$ substrate; (c) spectrum of the characteristic radiation of particles under electron-beam probing.

The indicated regularity is attributable to the process of smoothing out of the irregularities of the surface relief by a train of laser pulses. This fact, confirmed experimentally, must be taken into account with the aim of raising the efficiency of the method of laser synthesis of gold nanoparticles [6].

\section{EXPERIMENTAL RESULTS AND DISCUSSION}

\subsection{Formation of Gold Colloidal Solutions}

We can penetrate gold particles formed in the vapor-plasma plume into a liquid medium, thus obtaining a gold colloidal solution [5,7]. A fairly small mass of particles is generally produced by the single action of a laser pulse (the total ablative mass which transforms into particles of the condensed phase in the case of exposure to a single pulse is units to tens of micrograms depending on the exposure conditions) [6]. Therefore, for sufficiently concentrated gold colloidal solutions to be obtained, it is expedient to use consequences of acting laser pulses, which is quite simply available with today's laser equipment operating in the frequency regime. It's only necessary to provide the constant changing of localization of the laser focal spot on the target surface.

In the present work, we used an Nd:YAG laser $(\lambda=1064 \mathrm{~nm})$ with a pulse duration of $20 \mathrm{~ns}$ and an average pulse energy of $200 \mathrm{~mJ}$ to form aqueous colloidal solutions of gold; this enabled us to obtain a power density of $0.1-0.5 \mathrm{GW} / \mathrm{cm}^{2}$ in focusing to the spot with $d=2-3 \mathrm{~mm}$. A massive gold plate (999.9) was used as the target whose chemical homogeneity has been confirmed by a state certificate. The pulserepetition frequency was $5 \mathrm{~Hz}$. The characteristic exposure time selected for obtaining optically dense samples of gold-containing colloidal solutions was 10-15 min for each sample of volume $120 \mathrm{~mL}$. The process of laser synthesis occurred in an air medium to subsequently penetrate the formed gold nanoparticles into distilled water or another type of liquid.

Scanning electron microscopy (SEM) was applied to investigate the characteristics of gold nanoparticles after the corresponding preparation of the samples (vaporize liquid from colloid). The results of investigation of the samples using SEM (Fig. 2b) have shown the presence of nanosize particles with an average size of $\sim 50 \mathrm{~nm}$ and a variance of the particle-size distribution of $30 \%$, which is characteristic of condensation processes of droplet formation [5,7]. The spectrum of characteristic radiation of the particles (under their excitation by a sharply focused electron beam-electron probe) on the carbon substrate (Fig. 2c) points to the fact that the material of these particles corresponds to the material of the target, i.e., to gold. The presence of the peaks characteristic of carbon and oxygen in the figure is due to the type of substrate of the sample. The data obtained with absorption spectroscopy (curve 1, Fig. 2a) confirm the presence of the characteristic peak of SPR in the absorption spectrum of the sample. Its maximum is at $520 \mathrm{~nm}$, which points to the presence of nanoparticles with an average size of the order of $50 \mathrm{~nm}$ in the colloidal solution and a variance of the particle-size distribution of $\sim 30 \%$ (the data is shown in comparison with theoretical model of SPR band for mentioned above parameters-curve 2, Fig. 2a) [13, 14]. The optical density of the sample enables us to estimate the nanoparticle concentration in the solution at $10^{11}$ $10^{12} \mathrm{~cm}^{-3}$. Such gold colloidal solutions are great practical interest of catalyst chemistry [15], biology and medicine [16]. 

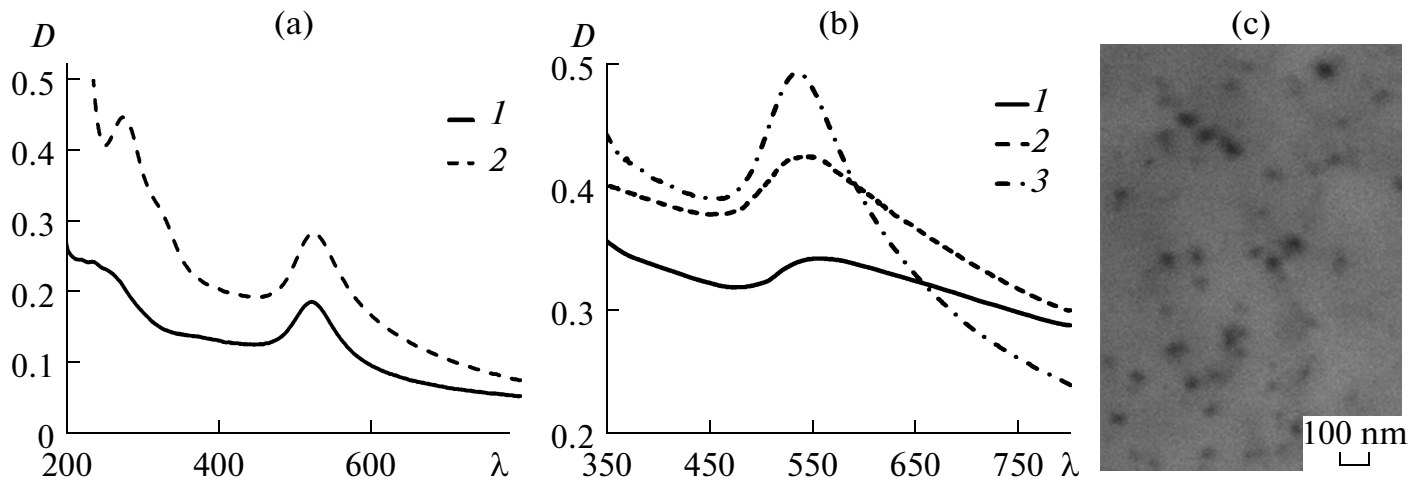

Fig. 3. The results of polymeric films containing golden nanoparticles investigation: (a) spectra of gold colloid ( 1 - pure, 2-with adding of 1\% PVA), (b) SPR band spectra (1-sample 1, 2-sample 2, 3-sample 3); (c) TEM image.

\subsection{Formation of Polymer Films Containing Gold Nanoparticles}

When solutions of film-forming substances are used as a penetration medium, after according processing a polymer films containing gold nanoparticles can be formed [17]. Low concentrations of film-forming substances $(1-3 \%)$ can not significantly change the physical characteristics of the penetration medium (water our case), which makes it possible to use the method described in the previous section unchanged. The typical spectrum of absorption of the aqueous of gold colloid, which contains $1 \%$ polyvinyl alcohol (PVA), is shown in Fig. 3a, curve 2: the slight "red" shift of the peak of plasmon resonance of gold (the maximum is at $525 \mathrm{~nm}$ ) is due to the surface-active action of PVA molecules (in comparison with pure water gold colloid-curve 1, Fig. 3a). At the same time, a pronounced absorption peak with its maximum at $273 \mathrm{~nm}$, which is characteristic of aqueous PVA solutions, is distinctly seen.

In the present work, under conditions identical to those described in the previous section, we formed aqueous colloidal solutions of gold nanoparticles, which contained, in low concentrations (1\%), the copolymer of acrylamide with sodium acrylate of composition 30/70 molar units and molecular weight $M_{n}=6 \times 10^{4}$ (sample 1), polymethyl methacrylamide with $M_{n}=8 \times 10^{3}$ (sample 2), and PVA (sample 3). Thereafter homogeneous films were formed from the samples by evaporating water at a temperature of $<50^{\circ} \mathrm{C}$.

It should be noted that the surface-plasmon-resonance band characteristic of gold is now noticeably shifted to the "red" region and its maximum is $570 \mathrm{~nm}$ for sample 1, $550 \mathrm{~nm}$ for sample 2, and $532 \mathrm{~nm}$ for sample 3 (Fig. 3b). This is due to the different degrees of influence of the surrounding polymer matrix on the surface electron density of a gold nanoparticle for different types of polymers. However, compared to the aqueous colloidal solutions, all the three samples are characterized by the effect of the "red" shift of the surface-plasmon-resonance band, which is due to the substantial redistribution of the electron density on the interface between the gold nanoparticles and the polymer matrix. Images of particles in the thin films of the polymers under study, obtained using transmission electron microscopy (TEM), are presented in Fig. 3c. Nanosize gold particles with characteristic dimensions of $\sim 50 \mathrm{~nm}$, which are quite uniformly distributed over the film's volume, are seen in the pictures.

Bimetallic samples can also be formed in the process of synthesis of colloidal solutions of metals upon a change in the kind of metallic target. For example, a sample of a PVA film containing simultaneously gold and silver particles was formed with the additional silver target [17]. The surface-plasmon-resonance band in this case has the characteristic two-peak form, with its maxima corresponding to silver (427 nm) [18] and gold (532 $\mathrm{nm})$.

The obtained polymer films can be used as non-luminescent light-power limiters in different optical devices. By applying different types of polymer matrix we can vary the location and form of SPR band to provide necessary optical properties [17].

\subsection{Formation of Gold Surface Island-Shaped Nanosructures}

To form the gold surface island-shaped nanostructures, we used the same Nd:YAG $(\lambda=1064 \mathrm{~nm})$ laser generating pulses of duration $20 \mathrm{~ns}$ with a mean energy of $200 \mathrm{~mJ}$, whose focusing into a spot with $d=2-$ $3 \mathrm{~mm}$ permitted obtaining a power density of $\sim 0.1-0.3 \mathrm{GW} / \mathrm{cm}^{2}$, with the pulse repetition frequency $5 \mathrm{~Hz}$. The characteristic exposure time for obtaining samples containing gold island-shaped nanostruc- 
(a)

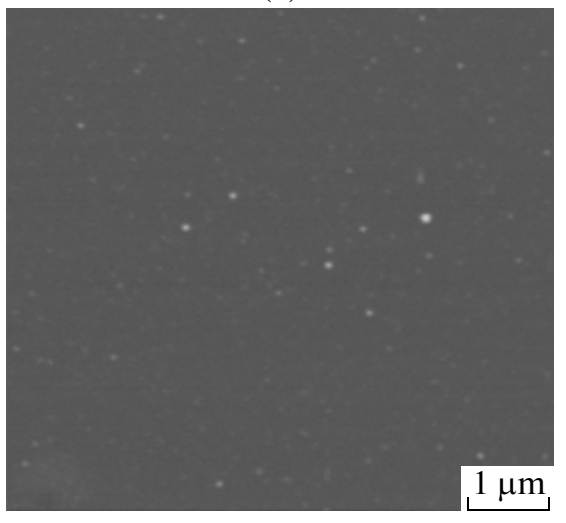

(c)

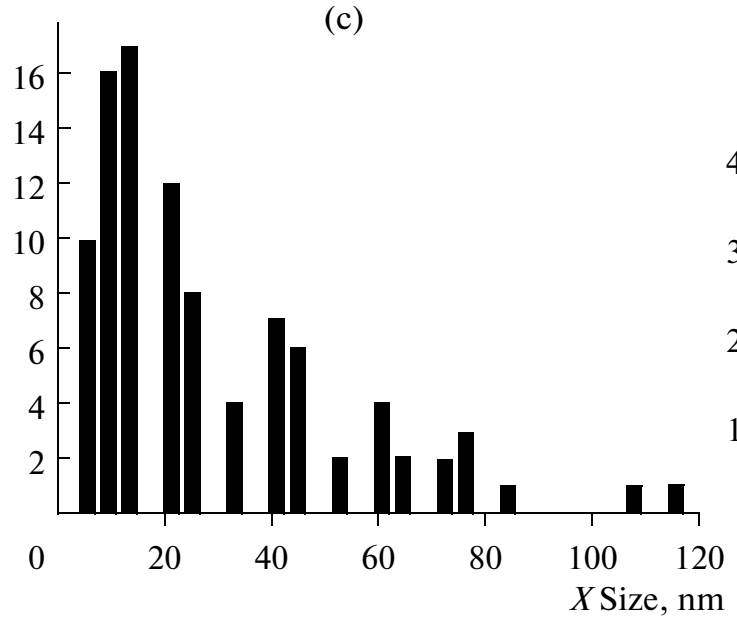

(b)

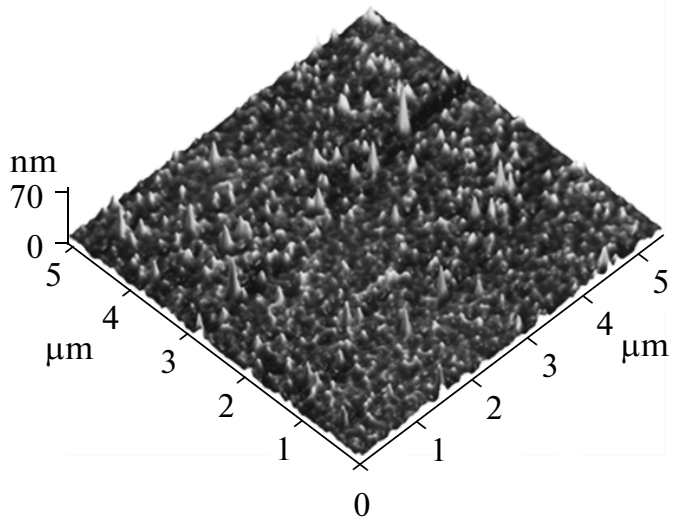

(d)

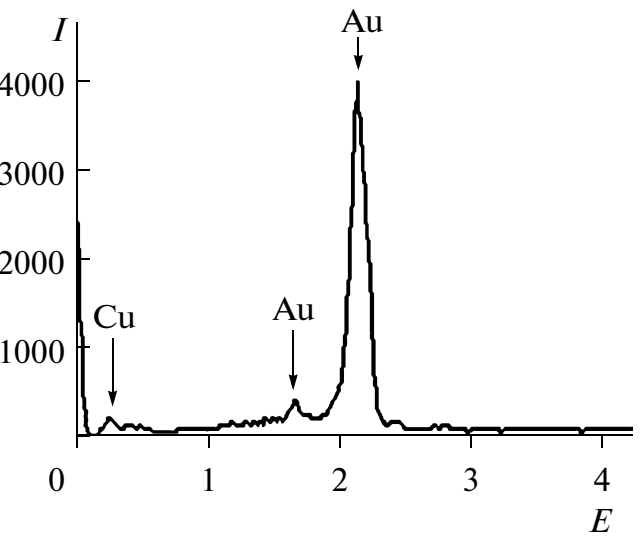

Fig. 4. The results of investigation of golden nanocones deposited on a Si substrate: (a) SEM image, (b) ASM image, (c) height distribution, (d) spectrum of the characteristic radiation of cones under electron-beam probing.

tures was chosen to be equal to 10-12 s (500-600 pulses) for one sample. The synthesis process proceeded in an air medium with subsequent deposition of gold nanostructures on silicon layer. For the target, we used massive plate from gold, which chemical homogeneity (999.9) has been confirmed by a state certificate. For the high effectiveness of laser erosion process the gradually changing of localization of the laser focal spot on the target surface was provided.

In this part of work, we used the following direct methods of diagnosing the parameters of the gold surface island-shaped nanostructures: SEM, atomic-force microscopy (AFM) and electron probe. The results of investigation of the samples using SEM (Fig. 4a) have shown the presence of island-shaped nanoobjects with roundish form of basement. Study of sample surface with the help of AFM shows the presence of vast amount of cone-shaped nanostructures (Fig. 4b) widely distributed with height (Fig. 4c). The spectrum of characteristic radiation of the particles on the silicon substrate (Fig. 4d) points to the fact that the material of these nanocones corresponds to the material of the target, i.e., to pure gold.

Such structures can find their practical applications in organic and polymer chemistry as heterogenic catalyst for appropriate reactions [15]; in biophysics as prosperous medium demonstrating giant Raman scattering effect [19] for investigation of complex molecules and cells; in the photo element industry for modification of photo element surface to enhance it sensibility in red and infra-red optical region on the base of SPR effect [20].

\section{CONCLUSIONS}

The industrial technology of formation of fluxes of nanosize gold particles at air can be based on the laser-synthesis method. The main advantages of this technology are simplicity of technological imple- 
mentation of the process, low production cost, and high rates of synthesis of gold nanostructures. Using water as the introduction medium, one can form aqueous colloidal solutions of gold with high indices of optical density. Introducing gold nanoparticles into the solutions of film-forming substances, one can obtain modified polymer materials having the absorption band due to the effect of SPR of the gold nanoparticles in the visible region. Samples with an intricate form of the absorption band can be formed with change in the type of targets in the process of synthesis. The obtained polymer films can be used as nonluminescent light-power limiters in different optical devices. On the base of air laser synthesis one can also form the gold surface island-shaped nanostructures, which can find their practical applications in organic and polymer chemistry, biophysics and the photo element industry. By virtue of the similarity of laser-erosion processes for different metals, the developed procedure will make it possible to form mention nanostructures of other noble metals.

\section{REFERENCES}

1. Tret'yakov, Yu.D., Microstructures of the New Functional Materials, Moscow: M.V. Lomonosov Moscow State University, 2006 [in Russian].

2. Raab, C., Simko, M., et al., Herstellung von Nanopartikeln und Nanomaterialien, Nanotrustdossiers, 2008, vol. 206, pp. 1-4.

3. Poole, Ch.P. and Owens, F.J., Introduction to Nanotechnology, London: John Wiley \& Sons, 2003.

4. Olenin, A.Yu. and Lisichkin, G.V., Metal nanoparticles in condensed media: preparation and the bulk and surface structural dynamics, Russ. Chem. Rev., 2011, vol. 80, pp. 605-630.

5. Goncharov, V.K., Kozadaev, K.V., and Shchegrikovich, D.V., Laser synthesis and investigation of the spectralmorphological characteristics of aqueous colloids of noble metals (Ag, Au, Pt), J. Eng. Phys. and Thermophys., 2012, vol. 85, pp. 788-793.

6. Goncharov, V.K., Kozadaev, K.V., et al., Formation of the condensed phase of metals exposed to intense nanosecond pulses at atmospheric pressure, J. Eng. Phys. and Thermophys., 2013, vol. 86, pp. 805-810.

7. Goncharov, V.K., Kozadaev, K.V., and Shchegrikovich, D.V., Investigation of noble metals colloidal systems formed by laser synthesis in air, Adv. Opt. Tech., 2012, 907292, doi: 10.1155/2012/907292

8. Ready, J.F., Effects of High-Power Laser Radiation, New York: Academic Press, 1971.

9. Palik, E.D., Handbook of Optical Constants of Solids, Orlando: Academic Press, 1985.

10. Goncharov, V.K., Kozadaev, K.V., et al., Occurrence of erosion processes in the near-surface region of metals exposed to intense nanosecond laser pulses, J. Eng. Phys. and Thermophys., 2013, vol. 86, pp. 798-804.

11. Goncharov, V.K., Kozadaev, K.V., and Shchegrikovich, D.V., Dynamics of the optical characteristics of erosion laser flares of metals formed by intense nanosecond laser pulses under atmospheric conditions, J. Appl. Spectr., 2013, vol. 80, pp. 395-402.

12. Kreibig, U. and Volmer, M., Optical Properties of Metal Clusters, USA: Springer, 1993.

13. Kozadaev, K.V., Diagnostics of aqueous colloids of noble metals by extinction modeling based on Mie theory, J. Appl. Spectr., 2011, vol. 78, pp. 692-697.

14. Goncharov, V.K. and Kozadaev, K.V., Complex optical method of express diagnostics of transparent media containing nanoparticles of noble metals, J. Eng. Phys. and Thermophys., 2013, vol. 86, pp. 868-874.

15. Astruc, D., Lu, F., and Aranzaes, J.R., Nanoparticles as recyclable catalyst: the frontier between homogeneous and heterogeneous catalysis, Angewandte Chem. Int. Ed., 2005, vol. 44, pp. 7852-7872.

16. Giliohann, D.A., Seferos, D.S., and Daniel, L., Gold nanoparticles for biology and medicine, Angewandte Chem. Int. Ed., 2010, vol. 49, pp. 3280-3294.

17. Goncharov, V.K., Kozadaev, K.V., et al., Formation and investigation of optical media containing gold nanoparticles, J. Eng. Phys. and Thermophys., 2012, vol. 85, pp. 42-47.

18. Goncharov, V.K., Kozadaev, K.V., and Shchegrikovich, D.V., Laser synthesis of optical media with silver nanoparticles by nanosecond at air, Optical memory \& Neural Networks (Information Optics), 2011, vol. 20, pp. 255259.

19. Nie, S. and Emory, S.R., Probing single molecules and single nanoparticles by surface-enhanced Raman scattering, Science, 1997, vol. 275, pp. 1102-1106.

20. Kozadaev, K.V., Laser synthesis of Ag island-shaped nanostructures at air, Proceedings of NAP, 2013, vol. 2, p. 03PISERE05. 